\title{
A Cognitive Approach to User Perception of Multimedia Quality: An Empirical Investigation
}

\author{
Sherry Y. Chen, Gheorghita Ghinea*, and Robert D. Macredie \\ School of Information Systems, Computing and Mathematics \\ Brunel University, Uxbridge, Middlesex, UB8 3 PH, UK. \\ e-mail: \{Sherry.Chen; George.Ghinea; Robert.Macredie\}@brunel.ac.uk
}

\begin{abstract}
Whilst multimedia technology has been one of the main contributing factors behind the Web's success, delivery of personalised multimedia content has been a desire seldom achieved in practice. Moreover, the perspective adopted is rarely viewed from a cognitive styles standpoint, notwithstanding the fact that they have significant effects on users' preferences with respect to the presentation of multimedia content. Indeed, research has thus far neglected to examine the effect of cognitive styles on users' subjective perceptions of multimedia quality. This paper aims to examine the relationships between users' cognitive styles, the multimedia Quality of Service delivered by the underlying network, and users' Quality of Perception (understood as both enjoyment and informational assimilation) associated with the viewed multimedia content. Results from the empirical study reported here show that all users, regardless of cognitive style, have higher levels of understanding of informational content in multimedia video clips (represented in our study by excerpts from television programmes) with weak dynamism, but that they enjoy moderately dynamic clips most. Additionally, multimedia content was found to significantly influence users' levels of understanding and enjoyment. Surprisingly, our study highlighted the fact that Bimodal users prefer to draw on visual sources for informational purposes, and that the presence of text in multimedia clips has a detrimental effect on the knowledge acquisition of all three cognitive style groups.
\end{abstract}

Keywords: Cognitive Style, Perceptual Quality, Quality of Service

* Corresponding author: phone +441895266033 ; fax +441895251686 


\section{Introduction}

The use of multimedia technologies in education is, by now, established. Whilst, by its very nature, research in this area has mostly concentrated on efficient integration of human factors in such technology [Kawalek, 1995; Schnotz, and Lowe, 2003; Wilson and Sasse, 2000; Yamazaki, 2001], outstanding issues still remain. Of these, in the present paper we shall specifically be concentrating our attention on two: quality and user cognitive style.

That we are concerned about quality in this context (and probably in many others) should not surprise, since quality considerations are important determinants behind the ultimate success or failure of multimedia educational applications. The issue is that, in practice, quality is a concept which, in the same context, varies according to the stakeholder group. In particular, when multimedia applications are distributed through the use of communication networks, quality considerations more often than not entail Quality of Service (QoS) parameters such as media loss rates, delays, or presentation frame rates, to name but a few. These parameters would mean little, however, to an end-user of a distributed multimedia application who is not technically-trained; nonetheless, if one were to ask such a user about the quality of the distributed multimedia application that (s)he had just been using, it is unlikely that one would not get a response in this respect. Indeed, some would argue that the end user's perception of quality is the deciding factor that eventually determines the success of such applications. To this end, in our research, we have characterised the user multimedia experience with the Quality of Perception (QoP) metric, which, in recognition of multimedia's infotainment duality (the property of multimedia applications to be located on the information-entertainment spectrum), not only deals with a person's subjective satisfaction with the quality of the multimedia application, but also his/her ability to understand, analyse and synthesise its informational content [Ghinea and Thomas, 1998].

The second dimension of our research deals with a user's cognitive style, namely his/her particular way of processing information [Jonassen and Grabowski, 1993]. Previous research has indicated that users with different cognitive styles prefer different ways to access information [Chen and Macredie, 2004]. Moreover, in a traditional, non-multimedia, learning environment, matching a user's cognitive style 
with content presentation has been shown to enhance his/her performance and improve perception [Ford and Chen, 2001]. Nonetheless, the influence of user cognitive style in distributed multimedia environments has remained a largely unexplored area of research. Within this context, we are particularly interested in quality issues - these are especially important because distributed multimedia is widely used in educational settings and findings from such investigations can be used to develop personalised distributed multimedia environments that accommodate individual differences.

Whilst in related work [Ghinea and Chen, 2006] of ours we have examined the interplay between QoP, QoS and user cognitive style, this study offered an incomplete picture of this interaction, as users were allowed to choose their QoS settings. In the current paper, we aim to provide more comprehensive evidence for the integration of quality and user cognitive styles in distributed multimedia environments, which examined the afore-mentioned interplay through the prism of a study in which users were shown multimedia content with a wide and evenly-distributed range of QoS settings.

Accordingly, the paper begins by building a theoretical background to present previous work in the area of subjective distributed multimedia quality and to discuss the influence of cognitive style on user perception of multimedia presentations. It then describes and discusses the findings of an empirical study that investigates the relationships between cognitive style and QoP. The paper ends with conclusions being drawn, highlighting the value of integrating QoP considerations with users' cognitive styles in the delivery of distributed multimedia presentations.

\section{Theoretical Background}

\subsection{Perceptual Impact of Quality of Service}

Traditional approaches to providing Quality of Service (QoS) to multimedia applications have focused on ways and means of ensuring and managing different technical parameters, such as delay, jitter and packet loss over unreliable networks. To a multimedia user, however, these parameters have little immediate meaning or impact. Although (s)he might be slightly annoyed at the lack of synchronisation 
between audio and video streams, it is unlikely that (s)he will notice, for instance, the loss of a single video frame out of the 25 which could be transmitted during a second of footage, especially if the multimedia video in question is one in which the difference between successive frames is small. Moreover, in a distributed setting, the underlying communication system will not be able to provide an optimum QoS owing to two competing factors multimedia data sizes and network bandwidth. This results in phenomena, such as congestion, packet loss, and errors, which have been extensively studied and reported upon in the literature [Nahrstedt and Steinmetz, 1995].

The effects of such artefacts on users and, more importantly, how to efficiently exploit them in multimedia communication technologies is an issue that has attracted relatively little attention, however. Indeed, much work in the Human-Computer Interaction field has concentrated exclusively on the application layer of the ISO/OSI communications model and has (optimistically) assumed that the underlying network subsystem is able to provide the QoS desired by the end-user [see, for example, Garrand, 1997; Hapeshi and Jones, 1992; Mayer, 1997; Reeves and Nass, 2000]. However, this is a best-case scenario, for in practice QoS fluctuations do and will occur, and it would be naïve to assume that they do not impact the user experience of multimedia-based learning in a distributed context.

In this respect, previous studies [Apteker et al., 1995; Fukuda et al., 1997; Hikichi et al., 2001; Wilson and Sasse, 2000; Yamazaki, 2001] exploring perceptual distributed multimedia quality and their integration across the layers of the ISO/OSI communications model can be characterised by two main observations: they concentrated almost exclusively on the entertainment dimension of multimedia (ignoring the informational aspect) and have highlighted the potential for significant resource savings to be made if perceptual considerations are integrated in the transmission of multimedia content.

One of the earliest experimental studies that investigated the impact on the user multimedia experience of a varying QoS factor (multimedia video frame rate) was undertaken by Apeteker et al. [1995]. They coined the term 'human receptivity' to 
mean not only how the human user perceives multimedia video shown at diverse frame rates, but also more distinct aspects of a user's acceptance of a video message. These include clarity and acceptability of audio signals, continuity of visual messages, lip synchronisation during speech, and the general relationship between visual and auditory message components. The most relevant result to come out of this work was that the dependency between human receptivity and the required bandwidth of multimedia clips is non-linear. Consequently, for certain ranges of human receptivity, a small variation leads to a much larger relative variation of the required bandwidth.

Closely related to this work is that of Fukuda et al. [1997] who derived a common mapping between the required bandwidth of multimedia video and three QoS parameters (frames per second, signal to noise ratio, and spatial resolution) independent of video content, whilst Yamazaki [2001] has examined the effects of different frame rates, sizes and quantization parameters of MPEG-4 video on subjective perceptual quality. Also working with MPEG-4 content, Cranley et al. [2003] investigated the use of perceived quality to deliver multimedia content over IP networks, while Hikichi et al. [2001] took a different perspective and explored the subjective effect of other QoS parameters such as delay, jitter and bandwidth in a networked haptic communication system, showing interest in perceptual channels other than vision. Without fail, all such research confirmed the fact that significant resource savings can be exploited in multimedia data transmission if perceptual considerations of quality were taken into account.

Human perceptual tolerance to media loss can also be exploited in the delivery of multimedia content. To this end, Wijesekera et al. [1999], in contrast to the work of Apteker et al [1995] and Fukuda et al [1997], which assumed that the underlying network communication system provided lossless multimedia streams, investigated the perceptual tolerance to discontinuity caused by media losses and repetitions, and to that of varying degrees of mis-synchronisation across streams. One of their initial results suggested that missing a few media units would not be negatively perceived by a user, as long as not too many such units were missed consecutively and that the occurrence was infrequent. Wijesekera et al. [1999] also found that media streams could drift in and out of synchronisation without causing considerable human 
annoyance. In their study, further evaluation of human tolerance to transient continuity and synchronisation losses with respect to audio and video showed that:

The pattern of user sensitivity varies depending on the type of media defect.

Viewer discontent for aggregate video losses increases gradually with the number of losses, while for other types of losses and synchronisation defects there is an initial sharp rise in viewer annoyance which afterwards plateaus out.

Video rate variations are tolerated much better than rate variations in audio.

Because human speech is characterised by talk periods interspersed with intervals of silence, audio loss in this case is tolerated quite well by humans as it results merely in silence elimination ( $21 \%$ audio loss did not provoke user discontent).

Whilst media synchronisation and loss are important (and omni-present, in today's distributed multimedia systems) factors which influence a user's subjective quality rating of multimedia presentations, the question also arises of what the cut-off rate is beyond which the quality of transmitted audio and video becomes unacceptable to human users. In a desktop conferencing environment, this issue has been explored by Kawalek [1995], who showed that the perception of media loss is highly taskdependent and that video losses are tolerated much better than audio ones. In related work, the impact of differing levels of streamed multimedia QoS has been examined for both standalone [Boring et al. 2002] and mobile systems [Song et al. 2002], while Bouch et al. [2000] have researched the effect of latency on perceived Web QoS. Indeed, the correlation between a user's subjective ratings of differing-quality multimedia presentations and physiological indicators has been studied by Wilson and Sasse [2000], whilst attempts to devise 'naturalistic' quality scales, based on a psychophysical modelling of subjective ratings, was the focus of work conducted by Boring et al. [2002] and Boring and Fernandes [2004].

User satisfaction, perception and understanding of multimedia should be the driving force in networking and operating systems research. Currently, research in these areas is driven from a purely technical perspective, with little or no analysis of the benefit to the user. The focus of our research has been on the enhancement of the traditional view of QoS with a user-level defined QoP. This is a measure which encompasses not 
only a user's satisfaction with multimedia clips, but also how the informational content of such presentations is perceived, synthesised and analysed.

As our review has highlighted, while the QoS impacts upon the perceived multimedia quality in distributed systems, previous work examining the influence of varying QoS on user perceptions of quality has almost totally neglected multimedia's infotainment quality and has concentrated primarily on the perceived entertainment value of presentations displayed with varying QoS parameters. We believe that a measure such as QoP, which specifically targets the infotainment aspect of multimedia, will have more meaning for a typical user than QoS metrics, and, in this paper, we explore the impact of individual differences, as given by the user's cognitive style (an individual's characteristic and consistent approach to organising and processing information [Weller et al., 1994]) on QoP.

\subsection{Cognitive Styles}

Cognitive styles influence how individuals prefer to organise and represent information [Riding and Rayner, 1998]. There are many dimensions of cognitive styles, among which Riding's [1991] Visualizer/Verbalizer particularly emphasises the presentation of information. Moreover, the preferences of Visualizers and Verbalizers gain particular importance [Clark and Paivio, 1991; Kirby et al., 1988; Mayer and Anderson, 1991; Paivio, 1990], since multimedia systems increasingly use novel ways of presenting information, such as through animation and video.

The Visualizer/Verbalizer style dimension is based on Dual Coding Theory proposed by Clark, Paivio (1991) and characterizes the inclination of an individual to represent information during thinking either through mental pictures or verbally. The main distinction between these two cognitive styles thus focuses on a preference for learning with words versus pictures [Jonassen and Grabowski, 1993]. Their differences are illustrated in Table 1.

As showed in Table 1, a Visualizer would prefer to receive information via graphics, pictures, and images, whereas a Verbalizers would prefer to process information in the form of words, either written or spoken [Jonassen and Grabowski, 1993]. In addition, Visualizers prefer to process information by seeing and they will learn most 
easily through visual and verbal presentations, rather than through an exclusively verbal medium. Moreover, their visual memory is much stronger than their verbal. On the other hand, Verbalizers prefer to process information through words and find they learn most easily by listening and talking [Laing, 2001]. A more detailed presentation of cognitive style classification is presented in section 3.3.2.

\begin{tabular}{|l|l|}
\hline Visualizers & Verbalizers \\
\hline Think concretely & Think abstractly \\
\hline $\begin{array}{l}\text { Have high imagery ability and vivid } \\
\text { daydreams }\end{array}$ & Have low imagery ability \\
\hline Like illustrations, diagrams, and charts & Like reading text or listening \\
\hline Prefer to be shown how to do something & Prefer to read about how to do something \\
\hline $\begin{array}{l}\text { Are more subjective about what they are } \\
\text { learning }\end{array}$ & $\begin{array}{l}\text { Are more objective about what they are } \\
\text { learning }\end{array}$ \\
\hline
\end{tabular}

Table 1. The Differences between Visualizers and Verbalizers [Adapted from Jonassen and Grabowski, 1993; Riding and Rayner, 1998]

The differences between Visualizers and Verbalizers are often not as great as some other cognitive styles. Indeed many Bimodal users are equally comfortable using either modality [Jonassen and Grabowski, 1993]. However, individuals appear to learn best when information can be readily translated into their preferred VerbalImagery mode of representation [Riding and Calvey, 1981]. Riding and Ashmore [1980] conducted an empirical study, which found that Verbalizers were superior with the verbal version, whilst Visualizers performed better in the pictorial mode. Another study by Riding and Sadler-Smith [1992] investigated the interaction between mode of presentation and style in their effect upon learning performance. Their study employed computer-based instructional materials in a variety of modes of presentation. They concluded that mode of presentation has important effects upon learning performance. Specifically, students on the Visualizer dimension improve most in learning due to the inclusion of more pictorial presentations about certain types of content.

Furthermore, Riding and Douglas [1993], with 15-16-year-old students, found that the computer-presentation of material on motorcar braking systems in a Text-plus-Picture format facilitated the learning by Visualizers compared with the same content in a 
Text-plus-Text version. They further found that in the recall task in the Text-plusPicture condition, $50 \%$ of the Visualizers used illustrations as part of their answers, compared to only $12 \%$ of the Verbalizers. Generally, Visualizers learn best from pictorial presentations, while Verbalizers learn best from verbal presentations. In another study by Riding and Watts [1997], there was a significant interaction between cognitive styles and the selection of presentation modes. The majority of Verbalizers selected a Verbal version and most of the Visualizers a Pictorial one. Other studies carried out by Riding, Buckle and Thompson [1989] and Riding and Anstey [1982] also returned similar findings.

The aforementioned studies have also indicated that further empirical work is needed to identify the preferences of such cognitive style groups. In particular, not enough work has been done to investigate the relationship between the use pattern of Visualizers and Verbalizers in multimedia systems in general, and specifically in distributed multimedia systems, where quality fluctuations can occur owing to dynamically varying network conditions. As the QoP metric is one which has an integrated view of user-perceived multimedia quality in such distributed systems, it is of particular interest to investigate the impact of cognitive styles on QoS-mediated QoP, as it will help in achieving a better understanding of the factors involved in such environments (distance learning and $\mathrm{CSCW}$, to name but two) and ultimately help in the elaboration of robust user models which could be used to develop applications that meet with individual needs.

Whilst in previous work of ours [Ghinea and Chen, 2006] we have explored the interplay between the two facets of quality, QoS and QoP, and cognitive style, this was done within the confines of a study in which users were free to choose their QoS settings (knowing that a choice of better QoS setting would penalise their QoP score). Moreover, the cognitive style dimension explored in this study was Field Dependency/ Independency. There is, however, a need to explore how this interplay is borne out when QoS is uniformly varied across participants, as is the need to explore a different dimension of cognitive style - whilst the Field Dependency/Independency dimension previously explored is mainly suited for user-controlled interactions, the Verbalizer/Visualizer dimension is particularly geared for system-controlled 
environments. This is precisely the scope of the study reported in this paper, whose methodological organisation we now proceed to describe.

\section{Methodology Design}

\subsection{Conceptual Framework}

This study investigates the impact of cognitive styles on perceived distributed multimedia quality. As multimedia information systems increasingly use visual technology, among a variety of cognitive styles, we examined the Verbalizer/Visualizer dimension in our research. Recognising that previous measures of perceived multimedia quality, such as human receptivity [Apteker et al. 1995], concentrate mainly on the entertainment/enjoyment aspect, our study used the QoP measure, the only such metric that takes into account multimedia's infotainment duality. Accordingly, Figure 1 presents the conceptual framework of this study - in a distributed multimedia context, applications are sent with various QoS parameters across networks such as the Web, which, depending upon the bandwidth available to such applications, impact upon their presentation quality, affecting parameters such as

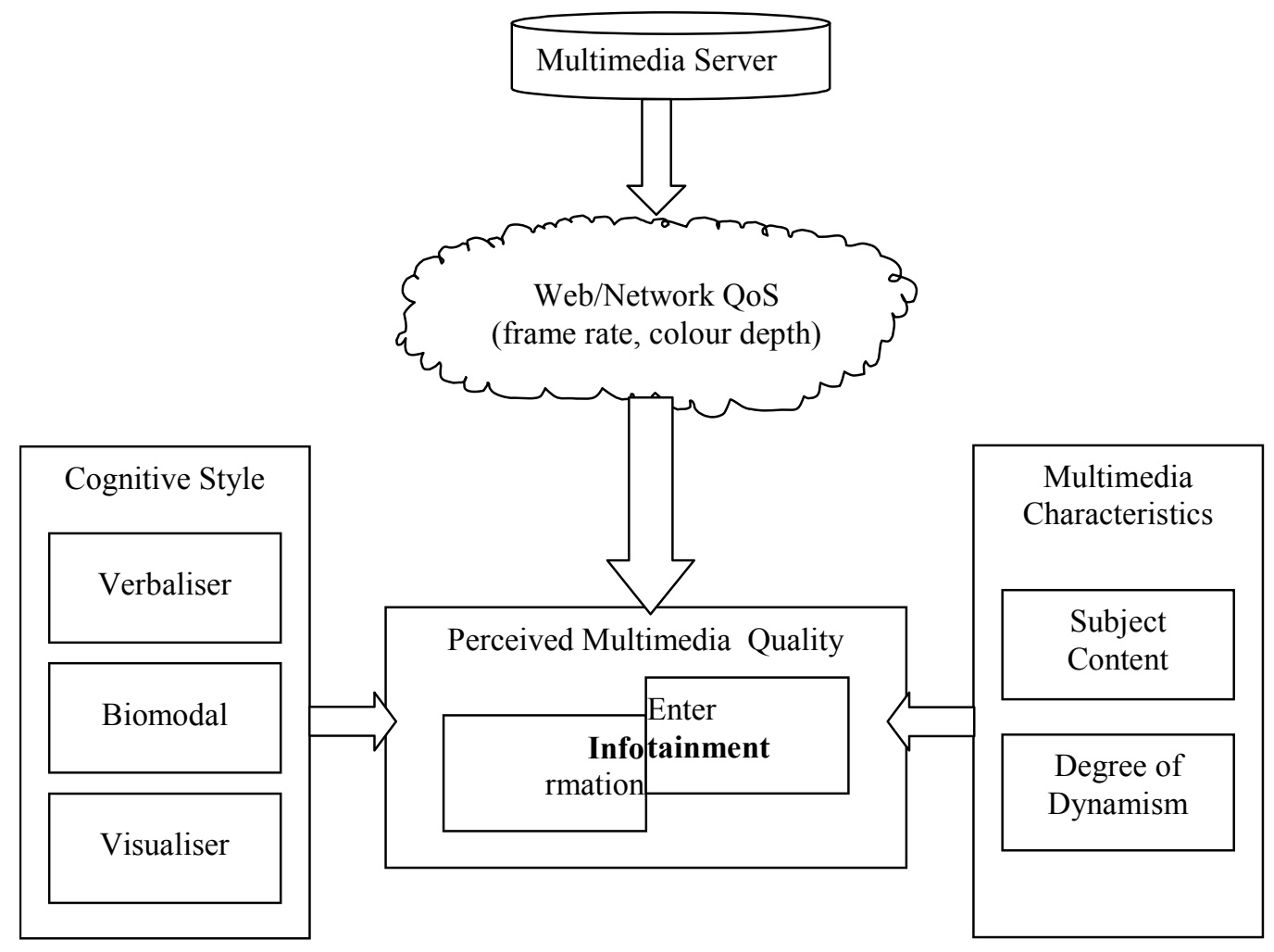

Figure 1: Conceptual Framework of this study 
possible display frame rates and colour depths. In turn, the perceived quality of these applications is potentially impacted by both the cognitive style of the user and the particular content of multimedia material (such as subject matter and degree of dynamism, i.e., of inter-frame variation).

In order to conduct a comprehensive evaluation, a wide range of independent variables are considered in this study. These are based on previous research in the area and include:

cognitive styles [Riding and Douglas, 1993], clip frame rate and colour depth [Apteker et al., 1995; Fukuda et al., 1997; Yamazaki, 2001], - these two variables are important determinants of multimedia data sizes and, by implication, of bandwidth (arguably the scarcest networking resource)

clip degree of dynamism [Apteker et al., 1995] - this variable is a good indicator of clip intra-frame redundancies (and, by implication of multimedia compression ratios and associated bandwidth transmission requirements); clip content - this variable characterises the differing subject matter of the experimental material.

The dependent variables of our study were the two components of Quality of Perception, QoP-IA and QoP-LoE. We employed a mixed design, which includes both between and within subject variables. The former are represented by cognitive style, clip frame rate and colour depth, while the latter include clip dynamism and content.

\subsection{Participants}

This study was conducted at Brunel University's Department of Information Systems and Computing. 132 subjects participated in the study. In spite of the fact that the participants volunteered to take part in the experiment, the breakdown according to gender resulted in equal male and female populations; however, the distribution according to cognitive styles was slightly uneven, as detailed in Table 2 . All of them 
were inexperienced in the content domain of the multimedia video clips visualized as part of our experiment, which will be described next.

\begin{tabular}{|l|r|r|r|}
\hline Cognitive Styles & \multicolumn{1}{|c|}{ Female } & \multicolumn{1}{|c|}{ Male } & \multicolumn{1}{c|}{ Total } \\
\hline \hline Verbalizer & 21 & 25 & 46 \\
\hline Bimodal & 17 & 15 & 32 \\
\hline Visualizer & 28 & 26 & 54 \\
\hline \hline Total & 66 & 66 & 132 \\
\hline
\end{tabular}

Table 2: The distribution of the sample

\subsection{Research Instruments}

\subsubsection{Apparatus}

All participants used the same IBM Thinkpad R40 laptop, with 512MB RAM and a 40GB hard drive, running the Microsoft Windows 2000 operating system on an Intel Pentium M 1.6GHz processor.

\subsubsection{Video Clips}

A total of 12 video clips were used in our study. The multimedia clips were visualized under a Microsoft Internet Explorer browser with a Microsoft Media player plug-in, with users subsequently filling in a Web-based questionnaire to evaluate QoP for each clip.

These 12 clips had been used in previous QoP experiments [Ghinea and Thomas, 1998], were between 30-44 seconds long, digitised in MPEG-1 format in a $352 * 288$ pixel window. The subject matter they portrayed was varied (as detailed in Table 3) and taken from selected television programs, thereby reflecting informational and entertainment sources that users might encounter in their everyday lives. The multimedia video clips used in this experiment were chosen to cover a broad spectrum of infotainment subject matter. Multimedia video clips vary in nature from those that are informational in nature (such as a news /weather broadcast) to ones that are usually viewed purely for entertainment purposes (such as an action sequence, a cartoon, a music clip or a sports event). Specific clips, such as the Cooking clip, were chosen as a mixture of the two viewing goals. Also varied was the dynamism of the clips (i.e., the rate of change between the frames of the clip), which ranged from a relatively static News clip to a highly dynamic Space Action movie. Table 3 also describes the importance, within the context of each clip, of the audio, video and 
textual components as purveyors of information, as previously established through user tests. These involved eight users rating four attributes (dynamism, video, audio and textual content) of a specific clip using 3 levels (weak, medium, strong). Intercoder reliability was high (86\%) and differences were settled by discussion. A brief characterisation of the clips now follows:

Action Movie clip - this is an action scene from a popular science fiction series. As is common in such sequences it involves rapid scene changes, with accompanying visual effects (explosions).

Animation clip - this clip features a disagreement between two main characters. Although dynamically limited, there are several subtle nuances in the clip, for example: the correspondence between the stormy weather and the argument.

Band clip - this shows a high school band playing a jazz tune against a background of multicoloured and changing lights.

Chorus clip - this clip presents a chorus comprising 11 members performing mediaeval English music. A digital watermark bearing the name of the TV channel over it is subtly embedded in the image all through the recording.

Commercial clip - an advertisement for a bathroom cleaner is being presented. The qualities of the product are praised in four ways - by the narrator, both through the audio and visually by the couple being shown in the commercial, and textually, through a slogan display.

Cooking clip - although largely static, there is a wealth of culinary information being passed on to the viewer. This is done both through the dialogue being pursued and visually, through the presentation of ingredients being used in cooking the meal.

Documentary clip - a feature on lions in India. Both audio and video streams are important, although there is no textual information present.

News clip - contains two main stories. One of them is presented purely by verbal means, while the other has some supporting video footage. Rudimentary textual information (channel name, newscaster's name) is also displayed at various stages.

Pop clip - is characterised by the unusual importance of the textual component, which details facts about the singer's life. From a visual viewpoint 
it is characterised by the fact that the clip was shot from a single camera position.

Rugby clip - presents a test match between England and New Zealand. Essential textual information (the score) is displayed in the upper left corner of the screen. The main event captured is the score of a try. As is expected, the clip is characterised by great dynamism.

Snooker clip - the lack of dynamism in this clip is in stark contrast to the Rugby clip. Textual information (the score and the names of the two players involved) is clearly displayed on the screen.

Weather clip - this is a clip about forthcoming weather in Europe and the UK. This information is presented through the three main modalities possible: visually (through the use of weather maps), textually (information regarding envisaged temperatures, visibility in foggy areas) and by the oral presentation of the forecaster.

\begin{tabular}{|c|c|c|c|c|}
\hline VIDEO CATEGORY & Dynamic & Audio & Video & Text \\
\hline 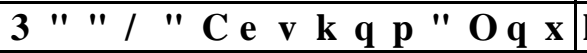 & L S Strong & Medium & Strong & Weak/None \\
\hline 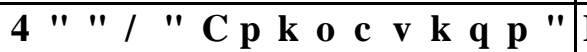 & \& Mddism & Medium & Strong & Weak/None \\
\hline 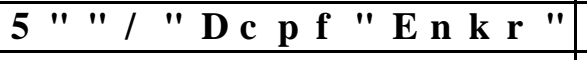 & Medium & Strong & Medium & Weak/None \\
\hline 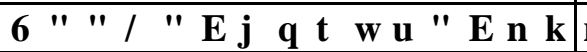 & S $\square$ Weak & Strong & Medium & Weak/None \\
\hline 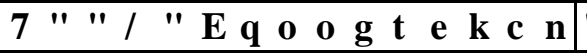 & 0 Radiluns & Strong & Strong & Medium \\
\hline 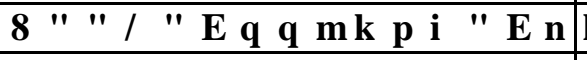 & L SWeak & Strong & Strong & Weak/None \\
\hline 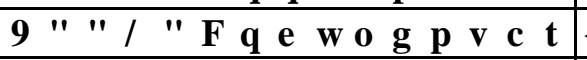 & I MediOnL S & Q Strong & Strong & Weak/None \\
\hline 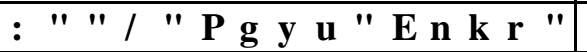 & \begin{tabular}{l|l} 
Weak &
\end{tabular} & Strong & Strong & Medium \\
\hline 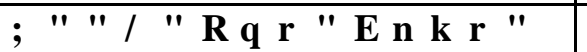 & Medium & Strong & Strong & Strong \\
\hline 10 - Rugby Clip & Strong & Medium & Strong & Medium \\
\hline 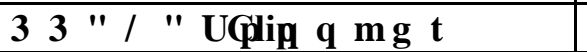 & Weak & Medium & Medium & Strong \\
\hline 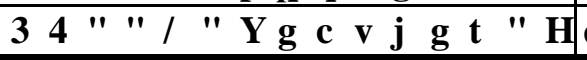 & UWE B D & & & Stron \\
\hline
\end{tabular}

Table 3 Video Categories Used in Experiments

\subsubsection{Cognitive Style Analysis}

The cognitive style dimension investigated in this study was Verbalizer/Visualizer. A number of instruments have been developed to measure this dimension. Riding's [1991] Cognitive Style Analysis (CSA) was applied to identify each participant's cognitive style in this study, because the CSA offers computerised administration and scoring. In addition, the CSA can offer various English versions, including Australasian, North American and UK contexts. The CSA uses two types of statement to measure the Verbal-Imagery dimension and asks participants to judge 
whether the statements are true or false. The first type of statement contains information about conceptual categories while the second describes the appearance of items.

There are 48 statements in total covering both types of statement. Each type of statement has an equal number of true statements and false statements. It is assumed that Visualizers respond more quickly to the appearance statements, because the objects can be readily represented as mental pictures and the information for the comparison can be obtained directly and rapidly from these images. In the case of the conceptual category items, it is assumed that Verbalizers have a shorter response time because the semantic conceptual category membership is verbally abstract in nature and cannot be represented in visual form. The computer records the response time to each statement and calculates the Verbal-Visualizer Ratio. A low ratio corresponds to a Verbalizer and a high ratio to a Visualizer, with the intermediate position being described as Bimodal. It may be noted that in this approach individuals have to read both the verbal and the imagery items so that reading ability and reading speed are controlled for. Table 4 illustrates the measurement of the Verbalizer/Visualizer ratio based on Riding' recommendation (1991). These recommendations were followed in this study.

\begin{tabular}{|l|l|}
\hline Ratio $<0.98$ & Verbalizer \\
\hline $0.98<$ Ratio $<1.09$ & Bimodal \\
\hline Ratio $>1.09$ & Visualizer \\
\hline
\end{tabular}

Table 4: Cognitive Style Categorisation according to the Verbalizer/Visualizer Ratio

\subsection{Measuring QoP}

As previously mentioned, QoP has two components: an information analysis, synthesis and assimilation part (henceforth denoted by $4 R \quad 3 \quad$ ) and $\$ a$ subjective level of enjoyment (henceforth denoted by $4 R 3 \square$ ). $\mathbb{R}$ (understand QoP in the context of our work, it is important to explain how both these components were defined and measured. 


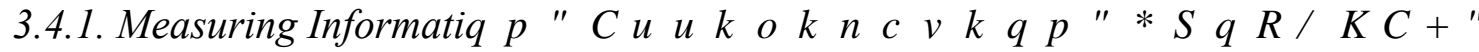

In our approach, QoP-IA was expressed as a percentage measure, which reflected a user's level of information assimilated from visualised multimedia content. Thus, after watching a particular multimedia clip, the user was asked a standard number of questions (10, in our case) which examined information being conveyed in the clip just seen, and QoP-IA was calculated as being the proportion of correct answers that users gave to these questions. All such questions asked must, of course, have definite answers, for example: (from the Rugby video clip used in our experiment) "What teams are playing?" had an unambiguous answer (England and New Zealand) which had been presented in the multimedia clip, and it was therefore possible to determine if a participant had answered this correctly or not. It must be noted that QoP-IA did not test just information recall, for quite a few questions could not be answered by recall of the clip content alone, but by the user making inferences and deductions from the information that had just been presented.

The composition of questions examining QoP-IA was determined through a pilot study which employed 10 participants. These sat experiments in which they answered a set of 14 questions per each multimedia clip. The purpose of this pilot study was to eliminate the two questions for which participants fared, on average, worst, or respectively, best, in terms of information assimilation, with the resulting 10 questions subsequently being used in the main study.

Since, in our experiment, questions could only be answered if certain information was assimilated from specific information sources (for example, the words of a song can only be gained from the audio stream), it is therefore possible to determine the percentage of correctly answered questions that relate to the different information sources within the multimedia video clip. Care was taken that information being examined was only conveyed through a single medium (for example, in a news cast, information that was conveyed both through audio and textual means, was not examined). For each feedback question the source of the answer was thus determined as having been assimilated from one of the following information sources:

$\mathrm{V}$ : Information relating specifically to the video window, for example, pertaining to the activity that lions in a documentary clip are engaged in.

A: Information which is presented in the audio stream. 
T: Textual information contained in the video window, for example: information contained in a caption.

Thus, by calculating the percentage of correctly absorbed information from different information sources, it was possible to determine from which information sources participants absorbed the most information. Using this data it is possible to determine and compare, over a range of multimedia content, potential differences that might exist in QoP-IA. The Cronbach coefficient for this measure was found to be 0.7574 , indicating a good reliability.

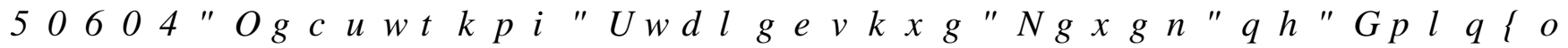
The subjective Level of Enjoyment (QoP-LoE) experienced by a user when watching a multimedia presentation was polled by asking users to express, on a scale of 1-6, how much they enjoyed the presentation (with scores of 1 and 6 respectively representing "no" and "absolute" user satisfaction with the multimedia video presentation).

In keeping with the methodology followed by Apteker et al [1995], users were instructed not to let personal bias towards the subject matter in the clip or productionrelated preferences (for instance the way in which movie cuts had been made) influence their enjoyment quality rating of a clip. Instead, they were asked to judge a clip's enjoyment quality by the degree to which they, the users, felt that they would be satisfied with a general purpose multimedia service of such quality. Users were told that factors which should influence their quality rating of a clip included clarity and acceptability of audio signals, lip synchronisation during speech, and the general relationship between visual and auditory message components. This information was also subsequently used to determine whether ability to assimilate information has any relation to user level of enjoyment, the second essential constituent (beside information analysis, synthesis and assimilation) of QoP. The Cronbach coefficient for this measure was found to be 0.7437 , again indicating good reliability

\subsection{Procedure}

The experiment consisted of several steps. Initially, the CSA was used to classify users' cognitive styles as Verbalizer, Bimodal or Visualizer. The participants then 
viewed the 12 multimedia video clips. Each video clip was shown with a specific set of QoS parameters, unknown to the user. In our experiment, only the video stream QoS was targeted, since it is the video component which consumes most bandwidth in multimedia applications, and bandwidth is the scarcest networking resource in such environments. Accordingly, we varied the frame rate with which presentations were shown (video clips were displayed at 5, 15 or 25 frames per second -fps) and the colour depth (which could either be full 24-bit colour or a black and white presentation). A total of 22 users for each (frame rate, colour depth) combination were tested in the experiment, with a relatively balanced distribution of cognitive styles across conditions, as depicted in Table 5.

\begin{tabular}{|l|l|l|l|}
\hline (frame_rate, colour_depth) & Verbalizer & Bimodal & Vizualiser \\
\hline (5fps, b/w) & 8 & 5 & 9 \\
\hline (15fps,b/w) & 9 & 6 & 7 \\
\hline (25fps, b/w) & 7 & 5 & 10 \\
\hline (5fps, 24bit) & 7 & 5 & 10 \\
\hline (15fps,24bit) & 8 & 6 & 8 \\
\hline (25fpd,24bit) & 7 & 5 & 10 \\
\hline
\end{tabular}

Table 5: Distribution of cognitive styles across conditions

In order to counteract any order effects, the order in which clips were visualised was varied randomly for each participant. After the users had seen each clip once, the window was closed, and the subjects had to answer a number of questions about the video clip that they had just seen in order to measure the QoP-IA and QoP-LoE. The user then went on and watched the next clip.

\subsection{Data Analyses}

Data were analysed with the Statistical Package for the Social Sciences (SPSS) for Windows version (release 9.0). An ANalysis Of VAriance (ANOVA), suitable to test the significant differences of three or more categories, and t-test, suitable to identify the differences between two categories [Stephen and Hornby, 1997], were applied to analyse the participants' responses. A significance level of $p<0.05$ was adopted for the study.

\section{Discussion of Results}

As mentioned in the preceding section, the dependent variables of our study were the two components of QoP. Accordingly, sections 4.1 and 4.2 look, from a cognitive 
style perspective, at the impact of clip categories and dynamism, respectively, on participants' QoP, whilst section 4.3 examines the influence of frame rate and colour depth on the same metric. Lastly, section 4.4 analyses the impact of cognitive styles on information assimilated from different media sources.

\subsection{Clip categories}

Our results indicate that clip categories, as given by their specific multimedia content matter, significantly influence participants' components of QoP-IA. This shows that the information assimilation scores are significantly influenced by the content being visualised; moreover, this observation is valid irrespective of the particular cognitive style of the participant. However, closer analysis reveals that different cognitive style groups have different favourite clips. Pop Music, which displays information using multiple channels, including video, audio, and text, is the favourite clip, from an information assimilation point of view, for Bimodals who combine the characteristics of both Verbalizers and Visualizers and are particularly adept at receiving information from either textual descriptions or graphic presentations.

\begin{tabular}{|l|c|c|c|}
\hline & Verbalizer & Bimodal & Visualizer \\
\hline Score & $62.84 \%$ & $62.98 \%$ & $65.52 \%$ \\
\hline & Documentary & \multicolumn{2}{|c|}{ Snooker } \\
\hline Enjoyment & 4.17 & 3.94 & 3.91 \\
\hline & Documentary & Pop Music & Documentary \\
\hline
\end{tabular}

Table 6: Favourite Clips

However, we did obtain some significant results that contradict those of previous research [Laing, 2001; Riding and Watts, 1997] (Tables 6 and 7). Although the Documentary clip does not display any text description, it is the clip in which, on average, Verbalizers obtain the highest QoP-IA $(\mathrm{F}=10.592$, df-within $=40, \mathrm{p}<.001)$. On the other hand, Visualizers perform better in the Snooker clip, which, though static, includes information conveyed through video, audio, and text $(\mathrm{F}=14.8451$, dfwithin=36, $\mathrm{p}<.001)$.

However, irrespective of cognitive style, we found that the Rugby clip was the one in which participants obtained the lowest QoP-IA scores $(\mathrm{F}=32.743$, df-within=72, $\mathrm{p}<.001$ ). Although this clip is similar in some respects to others studied by us (such as the Snooker clip, which also has an abundance of information being portrayed through video, audio and textual means), its main distinguishing feature is a high 
dynamism - there is considerable temporal variability due to the high inter-frame differences specific to clips featuring action sports. We therefore assume that the reason why participants scored so lowly in terms of QoP-IA on this clip is precisely because of its high dynamism, a hypothesis that shall be further explored in section 4.2.

\begin{tabular}{|l|c|c|c|}
\hline & Verbalizer & Bimodal & Visualizer \\
\hline Score & $35.59 \%$ & $35.74 \%$ & $34.49 \%$ \\
\hline & \multicolumn{3}{|c|}{ Rugby } \\
\hline Enjoyment & 2.59 & 2.78 & 2.81 \\
\hline & \multicolumn{3}{|c|}{ Rugby } \\
\hline
\end{tabular}

Table 7: Least Favourite Clip

Enjoyment will also influence users' performance, especially for Verbalizers, who perform better and enjoy more the Documentary clip and performed worse and enjoyed less the Rugby clip. This is consistent with the results of previous research [Chen, 2002], which highlight that positive user perceptions of the environment can enhance their performance; conversely negative attitudes will tend to hinder performance.

\subsection{Clip dynamism}

Analysis of the results obtained from the experiment shows that the degree of clip dynamism significantly impacts upon the QoP-IA component of QoP, irrespective of the user's cognitive style (Verbalizers: $F=6.359$; df-within $=549 ; \mathrm{p}=.002$; Visualizers: $\mathrm{F}=9.368$; df-within $=645 ; \mathrm{p}<.001$; Bimodals: $\mathrm{F}=8.217$; df-within $=381 ; \mathrm{p}<.001)$. The analysis has highlighted, moreover, the fact that the highest QoP-IA scores are obtained for clips which have a low degree of dynamism. Conversely, multimedia clips which have a high degree of dynamism have a negative impact on the user assimilation of the informational content being conveyed by the respective clips (Figure 2). Thus, clips which have relatively small interframe variability will facilitate higher QoP-IA scores: an object which might appear for only 0.5 seconds in a highly dynamic clip is less easily remembered than when it appears for one second in a clip which is less dynamic. 


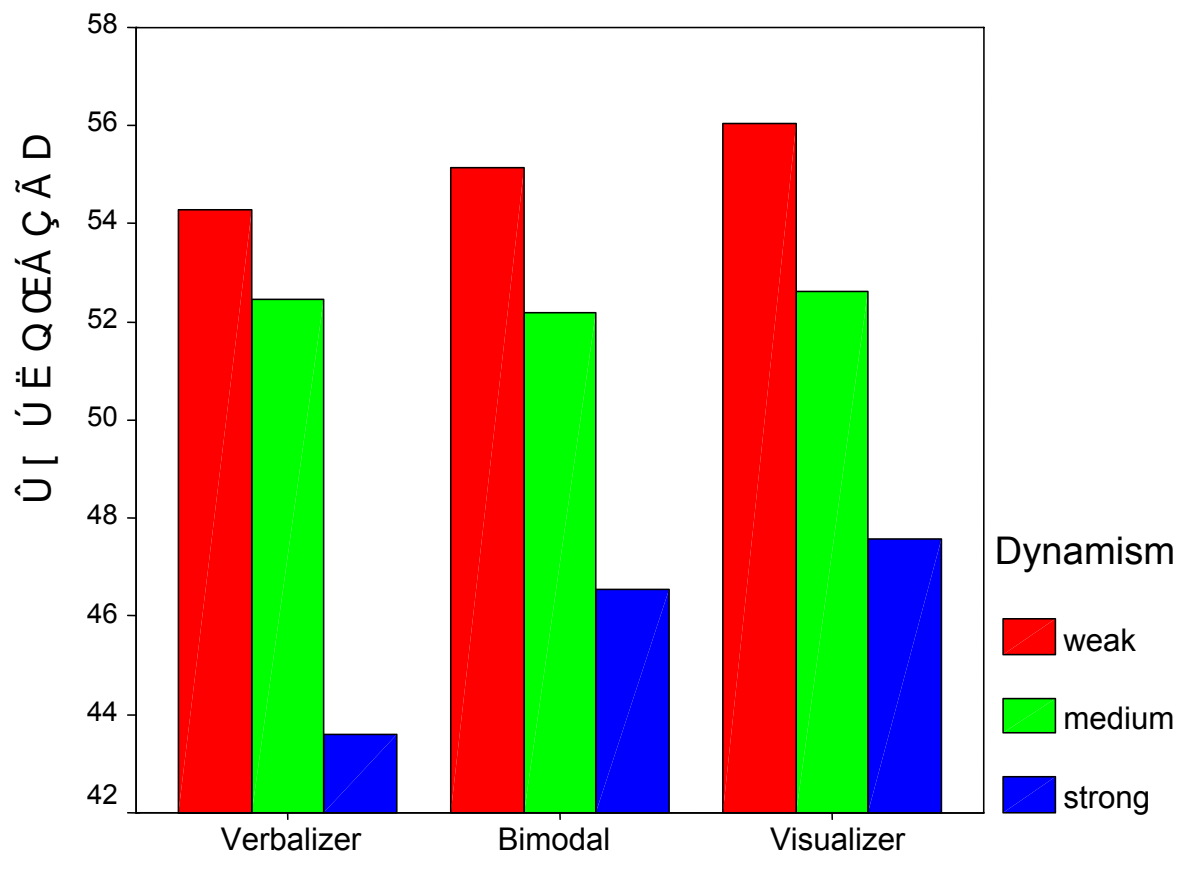

Cognitive Style

Figure 2: Cognitive Styles and Clip Dynamism Impact on QoP-IA

As far as the QoP-LoE component is concerned (Figure 3), analysis of our results reveals that whilst dynamism is a significant factor in the case of Verbalizers $(\mathrm{F}=8.009 ;$ df-within $=549 ; \mathrm{p}<.001)$ and Visualizers $(\mathrm{F}=4.691 ;$ df-within $=645$; $\mathrm{p}=.009)$, this is not true for Bimodals $(\mathrm{F}=2.824$; df-within $=381 ; \mathrm{p}=.061)$, although there is a trend toward significance for this cognitive style category as well. As suggested by previous research [Riding and Rayner, 1998], Bimodals can tailor learning strategies to the specific learning environments so the features of learning environments have no significant effects on their enjoyment. For Verbalizers and Visualizers, however, it was found that clips of medium dynamism had the highest levels of QoP-LoE, which suggests that such users do not find enjoyable clips which are static (or, conversely, highly dynamic). Whilst the user may feel somewhat overwhelmed by a fast-paced clip, (s)he might possibly feel uninterested by a static clip with (almost) repetitive frame displays; it should come as no surprise, then, that such users prefer clips of medium dynamism, where they do not feel overwhelmed, but neither are they bored by the presentation of the subject matter concerned. 


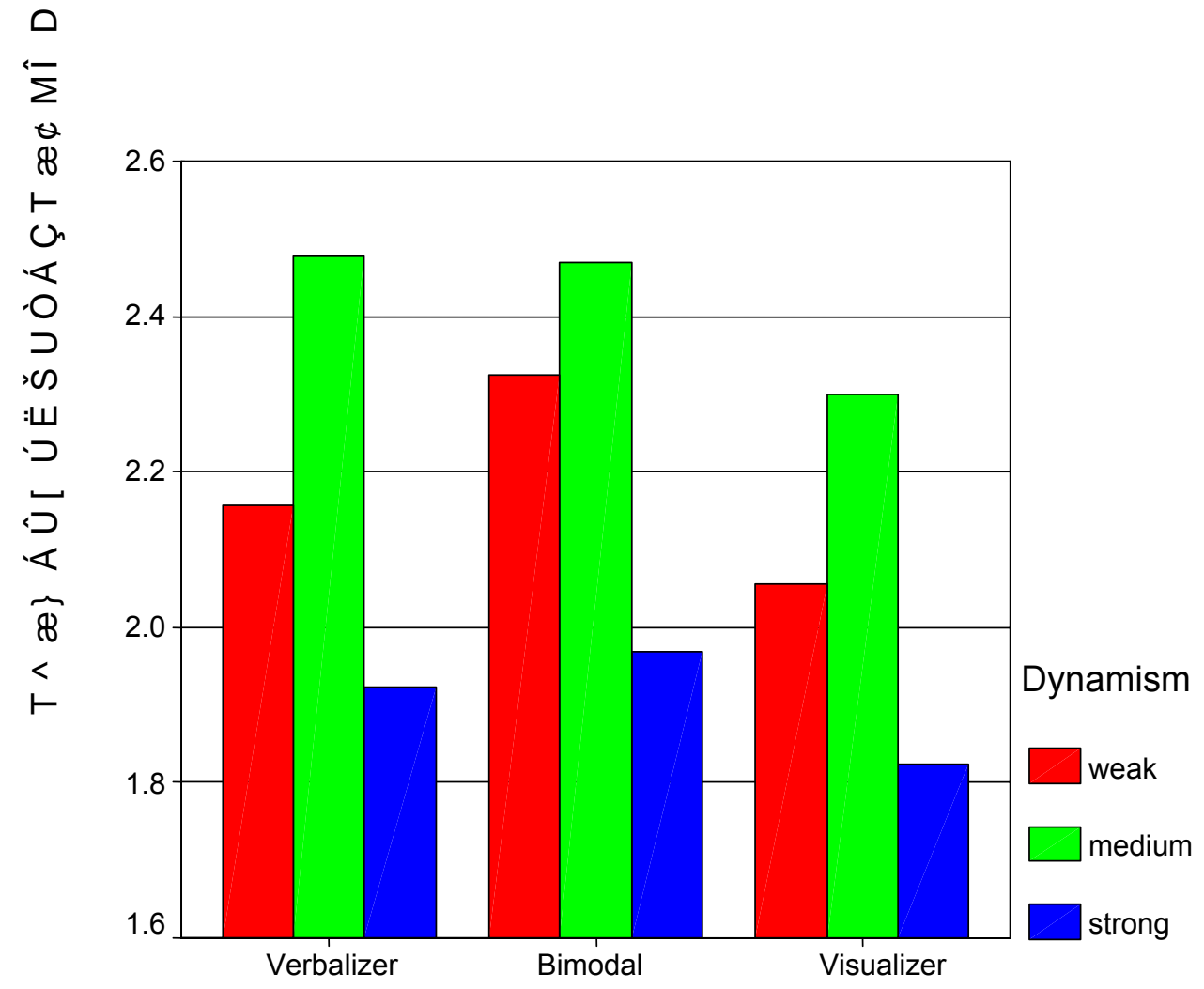

Cognitive Styles

Figure 3: Cognitive Styles and Clip Dynamism Impact on QoP-LoE

\subsection{Impact of Frame Rate and Colour Depth}

Our results indicate that the frame rates and colour depths with which the multimedia clips visualised as part of our experiment were presented do not significantly impact upon the two components of QoP (Figures 4-7). The fact that these two QoS parameters do not influence QoP is of particular importance in distributed, bandwidthlimited environments (such as those characteristic of wireless applications), as it suggests that user QoP is not negatively impacted by what are traditionally regarded as low (technical) quality presentations.

Additionally, the substantial bandwidth savings involved, even taking into account compression, in presenting a clip at $5 \mathrm{fps}$, with greyscale frames, instead of the full quality $25 \mathrm{fps}$, 24-bit colour depth, would mean that more multimedia sessions could be accommodated on a network. 


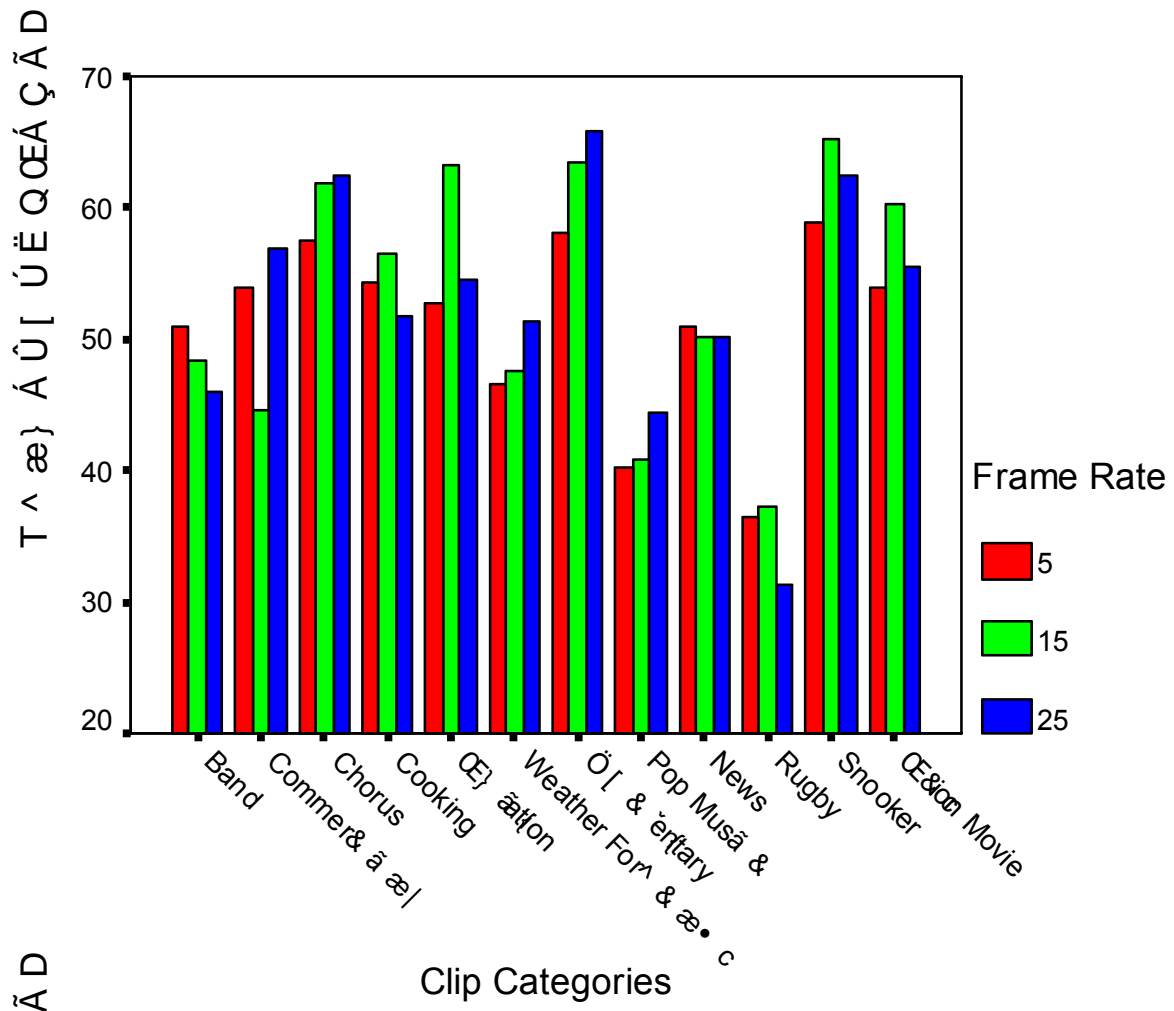

Figure 4: Frame Rate Impact on QoP-IA across Clip Categories

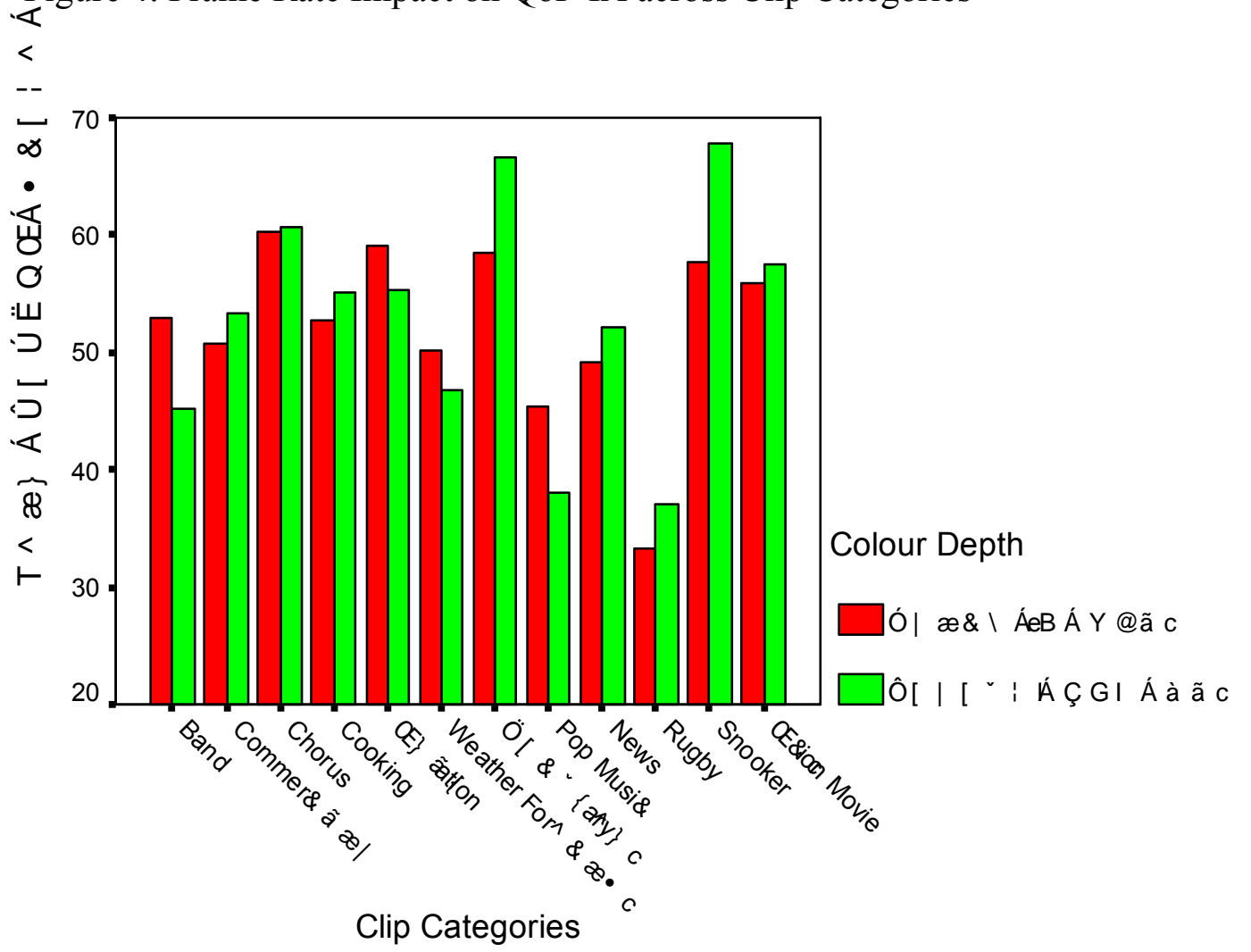

Figure 5: Colour Depth Impact on QoP-IA across Clip Categories 


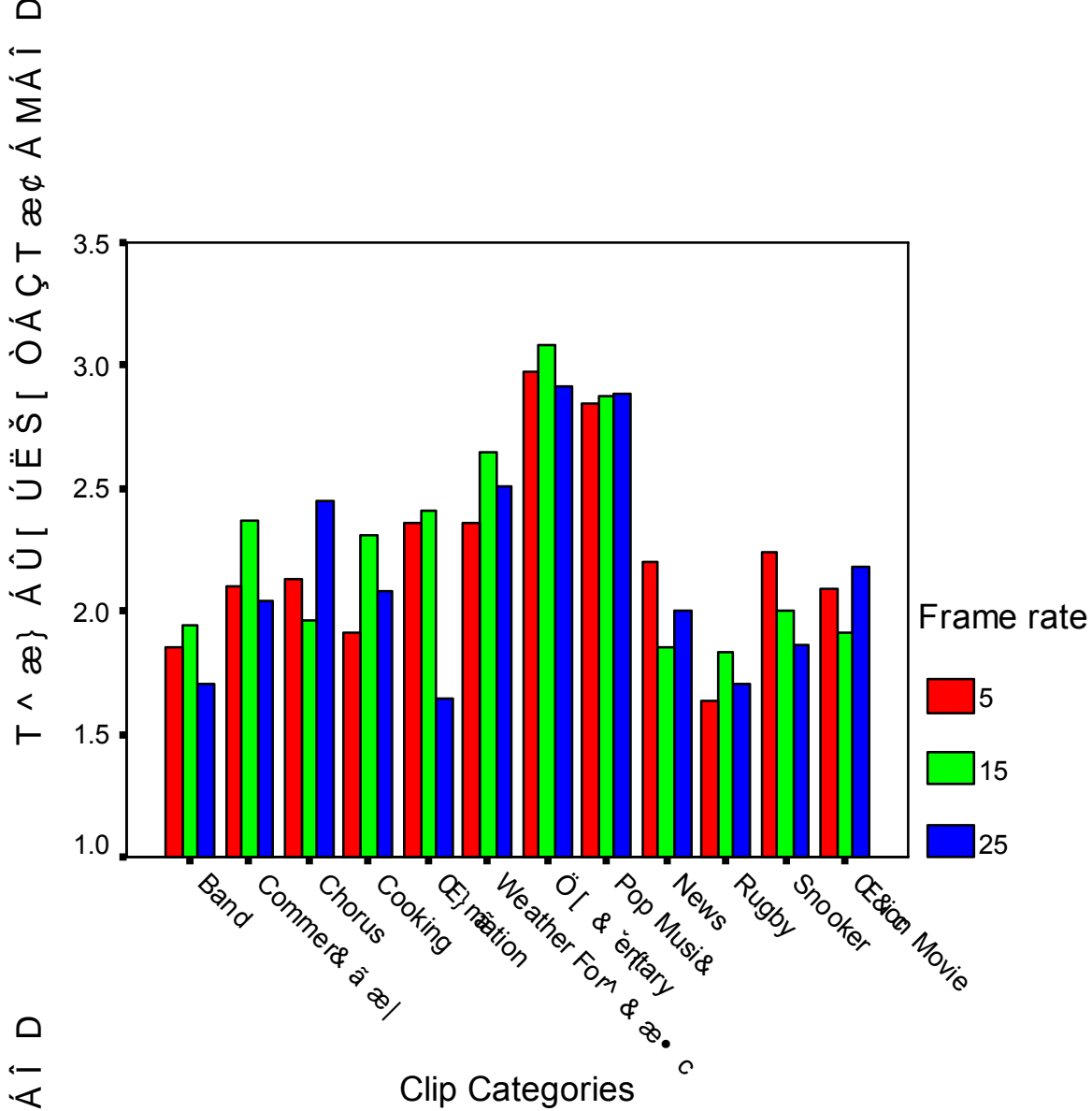

Figure 6: Frame Rate Impact on QoP-LoE across Clip Categories

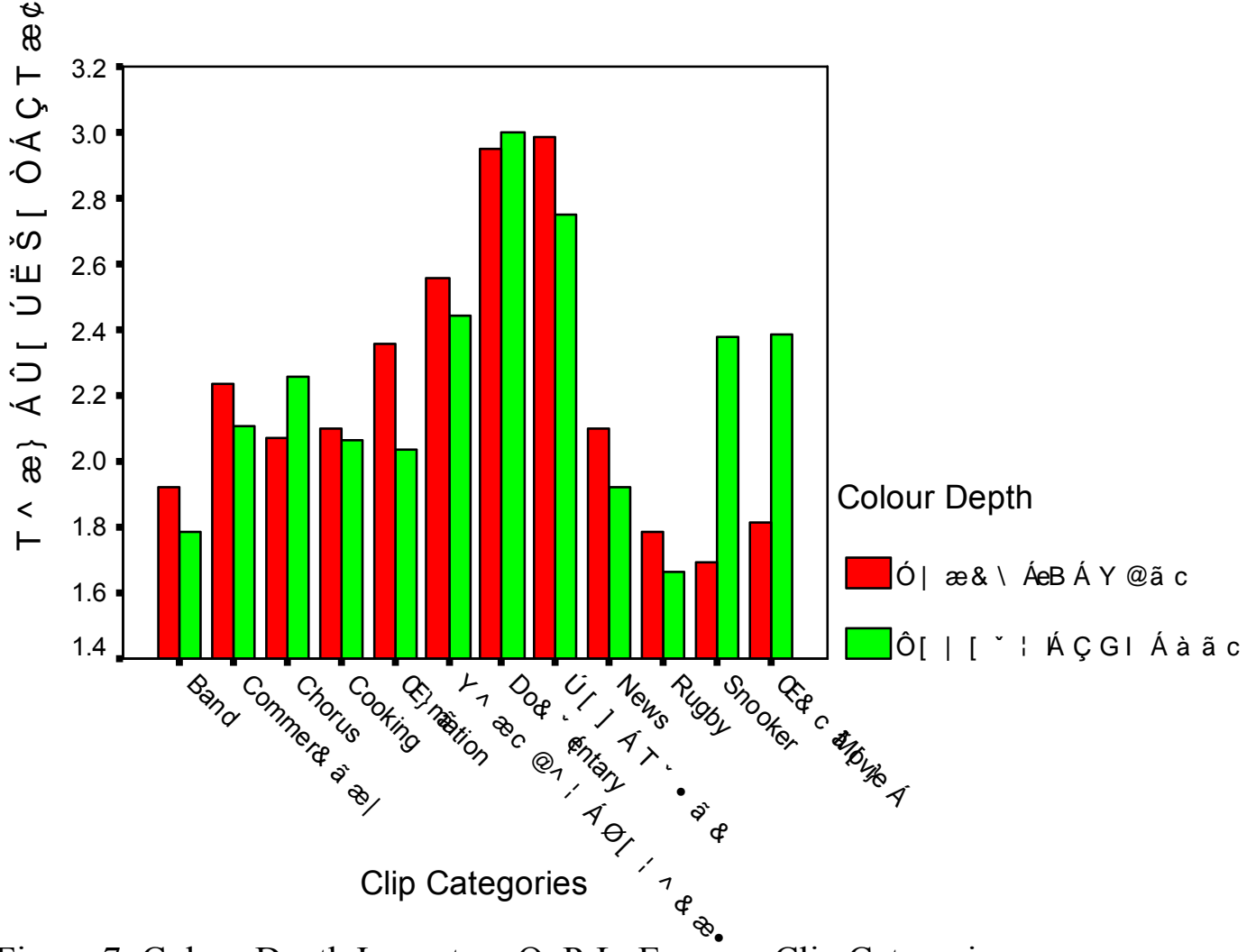

Figure 7: Colour Depth Impact on QoP-LoE acrotss Clip Categories 
It is worth noting that in contrast to previous findings [Apteker et al., 1995; Fukuda et al., 1997; Yamazaki, 2001] which showed that there was a dependency between human receptivity of multimedia applications and the frame rate with which they were viewed, our results would seem to indicate that when viewing a multimedia clip for educational/informational purposes as well, any degradations in quality are not noticed or are ignored by users. This suggests that perceived multimedia content is strongly related to context of use and the 'status' of informational content, and is in line with previous work, such as the findings of Kawalek [1995], who reported that users in a Computer Supported Co-operative Work Environment regard a video loss of $99 \%$ as 'still acceptable' if engaged in task solving.

\begin{tabular}{|l|l|l|l|}
\hline $\begin{array}{l}\text { Cognitive } \\
\text { Style }\end{array}$ & Effects & Frame Rate & Colour Depth \\
\hline Verbalizers & QoP-IA & $\begin{array}{l}\mathrm{F}=1.350 ; \text { df-between }=2 ; \\
\mathrm{p}=.260\end{array}$ & $\begin{array}{l}\mathrm{F}=3.652 ; \text { df-between }=1 ; \\
\mathrm{p}=.824\end{array}$ \\
\cline { 2 - 5 } & QoP-LoE & $\begin{array}{l}\mathrm{F}=.251 ; \text { df-between }=2 ; \\
\mathrm{p}=.778\end{array}$ & $\begin{array}{l}\mathrm{F}=.007 ; \text { df-between }=1 ; \\
\mathrm{p}=.902\end{array}$ \\
\hline Bimodal & QoP-IA & $\mathrm{F}=.315 ;$ df-between $=2 ;$ & $\mathrm{F}=.838 ;$ df-between $=1 ;$ \\
& & $\mathrm{p}=.730$ & $\mathrm{p}=.285$ \\
\cline { 2 - 5 } & QoP-LoE & $\mathrm{F}=.924 ;$ df-between $=2 ;$ & $\mathrm{F}=2.554 ;$ df-between $=1 ;$ \\
$\mathrm{p}=.396$ & $\mathrm{p}=.631$ \\
\hline Visualizers & QoP-IA & $\mathrm{F}=1.236 ; \mathrm{df}-$ between $=2 ;$ & $\mathrm{F}=.639 ;$ df-between $=1 ;$ \\
& & $\mathrm{p}=.291$ & $\mathrm{p}=.762 ;$ \\
\cline { 2 - 5 } & QoP-LoE & $\mathrm{F}=.886 ;$ df-between $=2 ;$ & $\mathrm{F}=.028 ;$ df-between $=1 ;$ \\
& & $\mathrm{p}=.413$ & $\mathrm{p}=.536$ \\
\hline
\end{tabular}

Table 8: Analysis of frame rate and colour depth impact on QoP

What is also interesting is that our results highlight that frame rates and colour depths have no significant effect on user QoP, irrespective of cognitive style (Table 8). These results (Figures 8-11) echo those of our previous study [Ghinea and Chen, 2006], which examined the three-way interaction between another dimension of cognitive style (Field Dependence), QoS parameters (frame rate and colour depth) and QoP. The results of that study also showed that the particular frame rates and colour depths with which a multimedia clip is being shown is not an important factor, from a QoP perspective, for each cognitive style group. Thus, the results of these two studies 
suggest that there is no close relationship between frame rate and colour depth, on the one hand, and user cognitive style, on the other. This finding in itself is interesting, for it highlights that bandwidth provision alone is not the key to providing perceptually better multimedia presentations, at least if the QoP metric is the one used to gauge perceptual multimedia quality.

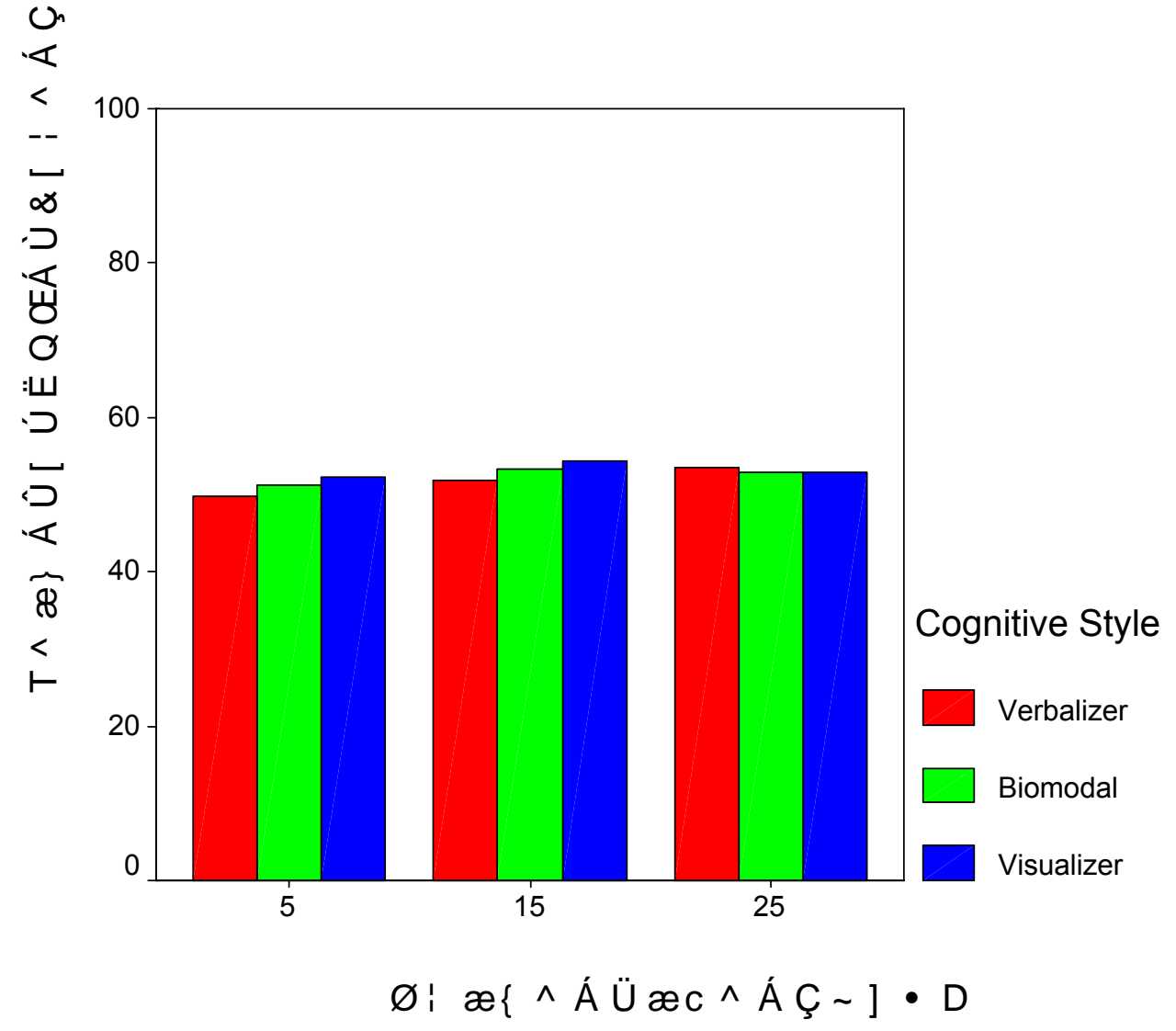

Figure 8: Impact of Frame Rates on QoP-IA according to Cognitive Styles 


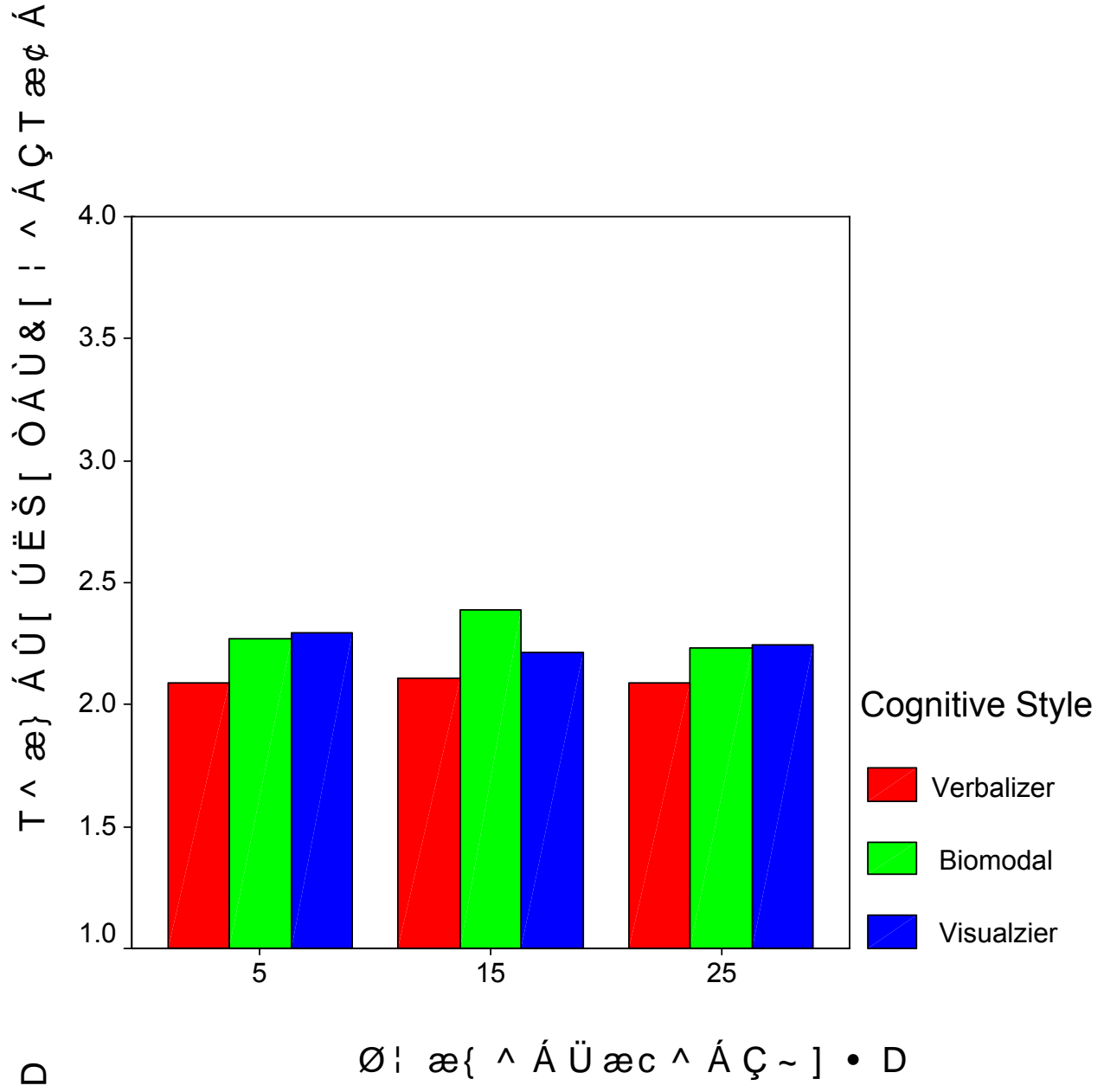

Figure 9: Impact of Frame Rates on QoP-LoE according to Cognitive Styles

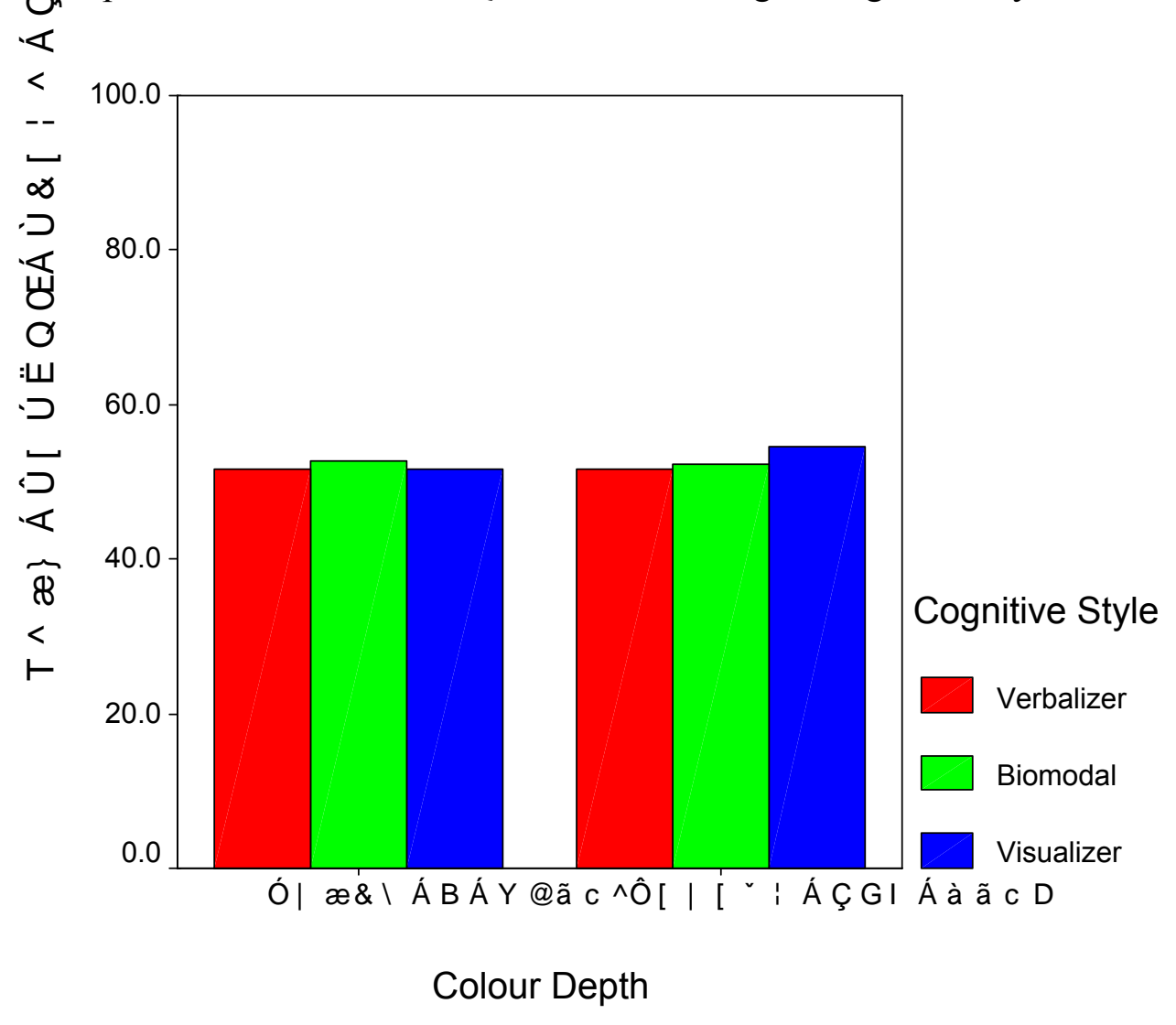

Figure 10: Impact of Colour Depth on QoP-IA according to Cognitive Styles 


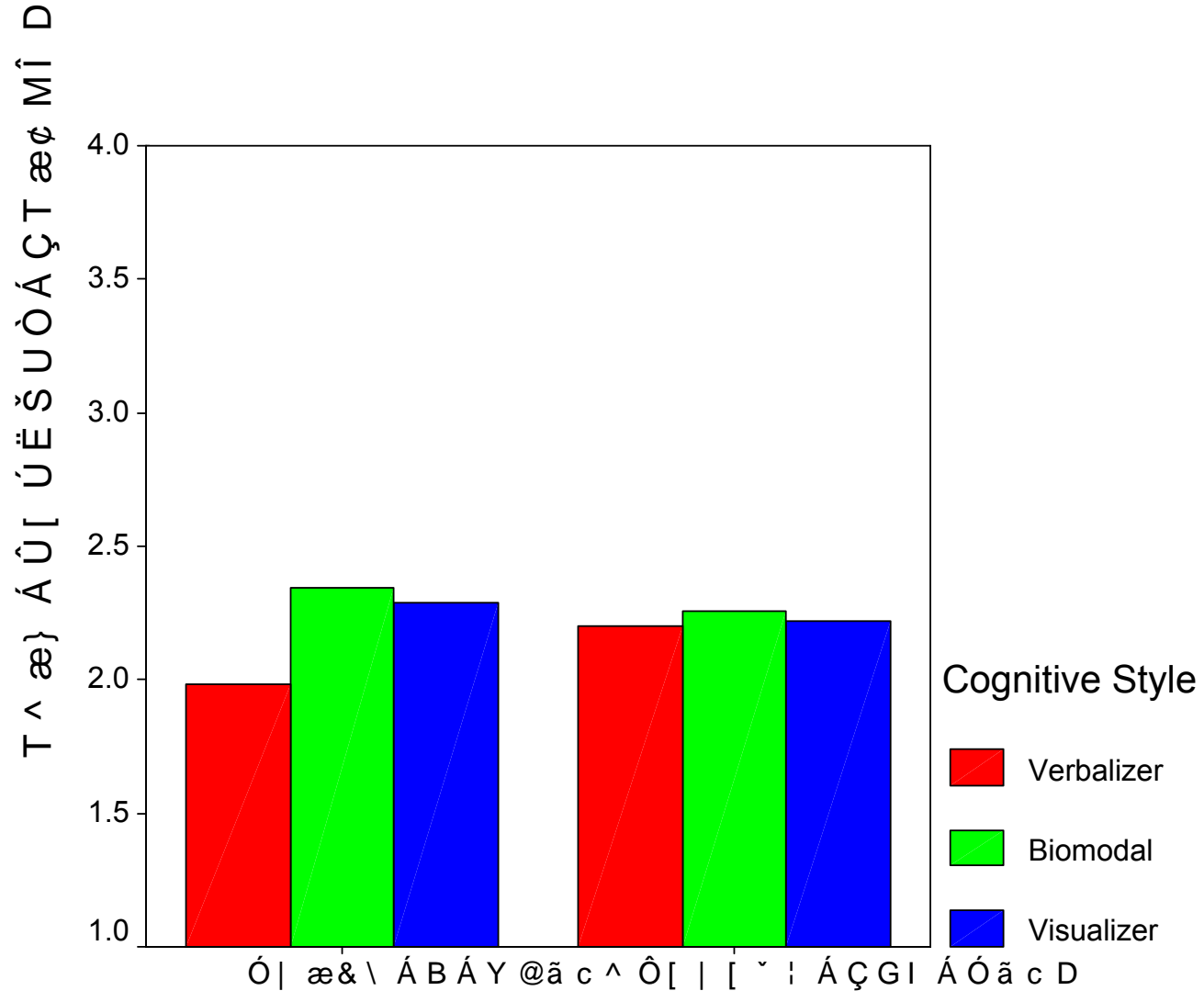

Colour Depth

Figure 11: Impact of Colour Depth on QoP-LoE according to Cognitive Styles

\subsection{Video vs. Audio vs. Text}

The particular cognitive style of a person may influence the QoP-IA score, according to whether the informational source lies in the video or audio component. We therefore also examined if the cognitive style impacts on how much information individuals assimilate from each of these two sources.

However, the ANOVA results showed that cognitive style seems not to be a significant factor in this case. In terms of video information, only one statistically significant result was found for the News clip $(F=4.101$; df-between $=2 ; \mathrm{p}=.021)$ clip. With regards to audio information, two statistically significant results were found in the case of the Weather Forecast $(\mathrm{F}=8.897$; df-between $=2 ; \mathrm{p}<.001)$ and News $(\mathrm{F}=4.250 ;$ df-between $=2 ; \mathrm{p}=.018)$ clips. On the other hand, for the six clips containing textual information in our study, it was found that Verbalizers obtain a statistically significant higher score than other cognitive styles, as shown in Table 9. 


\begin{tabular}{|l|c|c|}
\hline VIDEO CATEGORY & $\mathbf{F}$ & $\mathbf{p}$ \\
& (df-between $=2 ;)$ & \\
\hline Commercial Clip & 8.01 & .01 \\
\hline News Clip & 4.69 & .01 \\
\hline Pop Clip & 4.11 & .02 \\
\hline Rugby Clip & 3.13 & .05 \\
\hline Snooker Clip & 3.25 & .04 \\
\hline Weather Forecast Clip & 4.83 & .01 \\
\hline
\end{tabular}

Table 9: Textual information assimilation vs. Cognitive Style: ANOVA results

This last result is consistent with those of Riding and Douglas [1993] and Jonassen and Grabowski [1993], which showed that Verbalizers would prefer to process information in the form of text. However, the aforementioned result that there are mainly no statistical significant differences among three cognitive style groups for obtaining information from audio and video are different from those of Laing [2001] and Riding and Sadler-Smith [1992]. It may be due to the fact that these previous works presented different content formats separately, whilst ours presented different content formats at the same time, in a multimedia presentation. In other words, the former used a single channel to present information whereas the latter applied multiple channels to deliver content. This raises an interesting issue for future research to investigate whether different cognitive style groups have different preferences as regards single channels vs. multiple channels.

\section{Conclusion}

This paper has presented the results of an empirical study which examined the effect of cognitive styles on perceived distributed multimedia quality. Participants' cognitive styles were categorised as Verbalizers, Bimodal, and Visualizers by using Riding's CSA. Perceived multimedia quality was evaluated using the QoP metric, which encompasses not only a person's subjective satisfaction with the multimedia application (QoP-LoE), but also his/her ability to analyse, synthesise and assimilate its informational content (QoP-IA).

Our results show that, whilst multimedia video clip dynamism is an important factor impacting, irrespective of cognitive style, upon participants' QoP-IA levels, a similar 
conclusion as regards QoP-LoE can only be made with respect to Verbalizers and Visualizers. It has no significant effects on Bimodals, which, displaying characteristics of both Verbalizers and Visualizers, have adaptable preferences of accessing information and enjoy receiving information from multiple channels.

Frame rates and colour depths were shown not to significantly impact upon participants' QoP. Moreover, as this finding occurred irrespective of participants' cognitive styles, it emphasises that significant bandwidth savings can be made in distributed multimedia systems if one takes into account user perceptions of quality, since these do not decrease in line with degradations of multimedia technical quality.

This study has shown the importance of understanding of the interplay between cognitive styles and the two main quality facets, subjective and technical, of distributed multimedia applications. However, it was only a small step. Further studies need to be undertaken with a larger sample, and, ideally one in which each cognitive style has equal samples. Moreover, cognitive styles are only one aspect of personal characteristics that impact perceptions of work [Stewart and Barrick, 2003]. In the future, other human factors - such as gender differences, prior knowledge, or alternative construction of cognitive styles - could be examined in this context, as could a wider variety of multimedia content (for example, games, with a higher degree of interactivity). In addition, 'what users prefer' may be different from 'what is appropriate to users', so further research is needed to examine their differences in terms of cognitive styles. Such work can help to develop a better understanding of individual strategies used by different cognitive style groups so that designers can exploit the full potential of the QoP-QoS interplay and provide multimedia presentations with an enhanced QoP. The ultimate goal of such an understanding is to build robust user models for the development of personalised distributed multimedia environments and to integrate users' individual differences into truly end-to-end communication architectures.

\section{References}

Apteker, R.T., Fisher, J.A., Kisimov, V.S., and Neishlos, H. 1995. Video

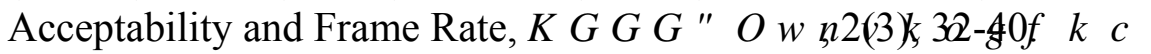


Boring, R.L., West, R.L., and Moore, S. 2002. Helping users determine video quality of service settings, Proceedings CHI '02, 598-599, Minneapolis, Minnesota.

Boring, R.L. and Fernandes, G.J. 2004. Measuring visual appeal of web pages, Proceedings CHI '04, 1557, Vienna, Austria.

Bouch, A., Kuchinsky, A., and Bhatti, N. 2000. Quality is in the eye of the beholder, Proceedings of the CHI 2000 Conference on Human Factors in Computing Systems, 297-304, The Hague, The Netherlands.

Chen, S. Y. and Angelides, M. C. (2003) Customisation of Internet multimedia information systems design through user modelling, in: Architectural Issues of

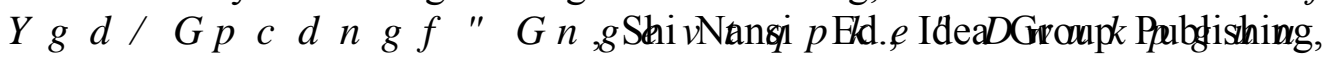
241-255.

Clark, J.M. and Paivio, A. 1991, Dual coding theory and education. ( $G X F D W L R Q D O$ Psychology Review, 71, 64-73

Cranley, N., Murphy, L. and Perry, P. 2003, User-perceived quality-aware adaptive delivery of MPEG-4 content, Proceedings of the 13th international workshop on Network and operating systems support for digital audio and video, 42-49, Monterey, CA.

Fukuda, K., Wakamiya, N., Murata, M., and Miyahara, H. 1997. QoS Mapping between User's Preference and Bandwidth Control for Video Transport, Proceedings of the 5th International Workshop on QoS (IWQoS), New York, USA.

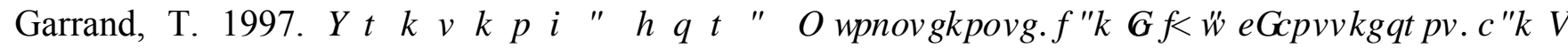
Advertising and the World Wide Web, Focal Press, Boston.

Ghinea, G. and Chen, S. Y. 2006. Perceived Quality of Multimedia Educational Content: A Cognitive Style Approach. ACM Multimedia Systems Journal. 11(3), 271-279.

Ghinea, G. and Thomas, J.P. 1998. QoS Impact on User Perception and Understanding of Multimedia Video Clips, Proceedings of ACM Multimedia '98, 49 - 54, Bristol, U.K.

Hapeshi, K. and Jones, D. 1992. Interactive Multimedia for Instruction: A Cognitive Analysis of the Role of Audition and Vision, , QWHUQDWLR QDO - RXUQDO Computer Interaction, 4(1), 79-99.

Hikichi, K., Morino, H., Matsumoto, S., Yasuda, Y., Arimoto, I., Ijume, M. and Sezaki, K. 2001. Architecture of Haptics Communication System for

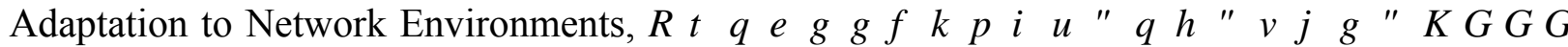

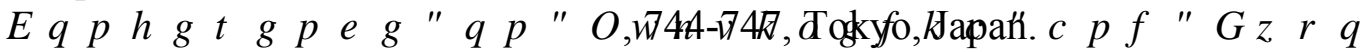

Jonassen, D. H. and Grabowski, B. 1993. Individual Differences and Instruction. New York: Allen \& Bacon. 
Kawalek, J. 1995. A User Perspective for QoS Management, Proceedings of the QoS Workshop aligned with the 3rd International Conference on Intelligence in Broadband Services and Network (IS\&N 95), Crete, Greece.

Kirby, J. R., Moore, P. J. and Schofield, N. J. 1988. Verbal and visual learning styles.

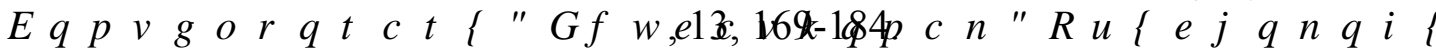

Laing, M. 2001 Teaching Learning and Learning Teaching: An Introduction to

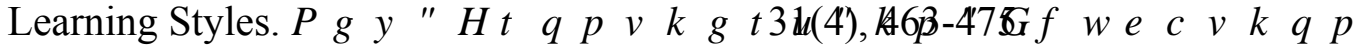

Mayer, R.E. 1997. Multimedia Learning: Are We Asking the Right Questions?,

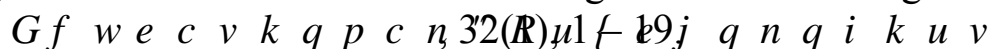

Mayer, R. E. and Anderson, R. B. 1991 Animations need narrations: An experimental

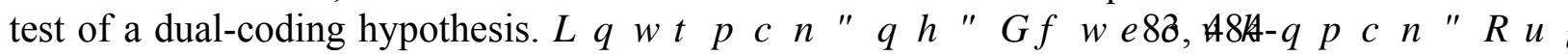
490.

Nahrstedt, K. and Steinmetz, R. (1995) Resource Management in Multimedia Networked Systems, , ( ( ( $\square \& R \$, \$ 2 \mp 64 W H U$

Paivio, A. 1990. Mental Representations: A Dual Coding Approach. Oxford: Oxford University Press.

Pask, G. 1976. Styles and strategies of learning, \%ULWLVK - RXUQDOQRI ( Psycholog, 46, 128-48.

Reeves, B. and Nass, C. 2000. Perceptual user interfaces: perceptual bandwidth, Communications of the ACM, 23(3), 65-70.

Riding R.J. and Douglas, G. 1993. The effect of cognitive style and mode of presentation on learning performance, \%ULWLVKप-RXUQDOQRI ( $G X F D$ Psychology, 63, 297-307.

Riding, R. J. 1991. Cognitive Styles Analysis, Birmingham: Learning and Training Technology.

Riding, R.J. and Anstey, L. 1982. Verbal-imagery learning style and reading attachment in eight year old children. Journal of Research in Reading 5, 57 66.

Riding, R.J. and Ashmore, J. 1980. Verbalizer-Imager Learning style and children's recall of information presented in pictorial versus written form, ( GXFDWLR QDO Studies, 6(2), 141-145.

Riding, R.J. and Calvey, I. 1981. The assessment of verbal-imagery learning styles and their effect on the recall of concrete and abstract prose passages by eleven year old children, British Journal of Psychology, 72, 59-64.

Riding, R.J. and Sadler-Smith, E. 1992. Type of instructional material, cognitive style and learning performance, ( $G X F D W L R Q 1 B$, B2B-\$64OWX GL HV 
Riding, R.J. and Watts, M. 1997. The effect of cognitive style on the preferred format of instructional material, ( $G X F D W L R Q D Q 7(13 \& 12)$, 179K1R3O R $J \quad \square$

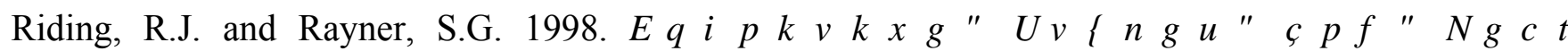
London: David Fulton Publisher.

Riding, R.J., Buckle, C., Thompson, S. and Hagger, E. 1989. The computer determination of learning styles as an aid to individualised computer-based

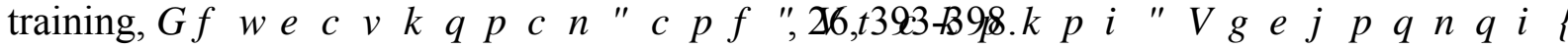

Schnotz, W. and Lowe, R. 2003. External and internal representations in multimedia learning, / HDUQL Q J D Q16, 1,7-Q2Y. WUX F WL R Q

Song, S., Won, Y. and Song, I. 2002. Empirical study of user perception behavior for mobile streaming, Proceedings of the tenth ACM international conference on Multimedia, 327-330, Juan-les-Pins, France.

Stephen, P. and Hornby, S. 1997. 6 L P S OH口 6 WDWL VWLFV F I R U I / $L E U$ Professionals. London: Library Association.

Stewart, G. L. and Barrick, M. R. 2003. Lessons learned from the person-situation debate: A review and research agenda. B. Smith \& B. Schneider (Eds.), Personality and Organizations. Lawrence Erlbaum Associates, Inc

Weller, H. G., Repman, J., and Rooze, G. E. 1994. The Relationship of learning, behavior, and cognitive styles in hypermedia-based instruction: Implications for design of HBI. Computers in the Schools, 10, 401-420.

Wijesekera, D., Srivastava, J, Nerode, A. and Foresti, M. 1999. Experimental Evaluation of Loss Perception in Continuous Media, Multimedia Systems, 7(6), 486-499.

Wilson, G. M. and Sasse, M. A. 2000. Investigating the Impact of Audio Degradations on Users: Subjective vs. Objective Assessment Methods. Proceedings of OZCHI'2000, Sydney, Australia, 135-142.

Yamazaki, T. 2001. Subjective Video Assessment for Adaptive Quality of Service

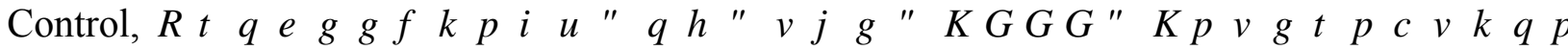
$D Q G \square,(517-52 R$, Tokyo, Japan. 